\title{
Controle biológico conservativo: Plantas herbáceas e a diversidade e abundância de dermápteros em algodoeiro colorido
}

\author{
João Marcos Amario de Sousa ${ }^{\bowtie}$ \& Danilo Henrique da Matta ${ }^{2}$
}

1. IFTO Campus Araguatins. 2. Sem vínculo institucional.

\section{EntomoBrasilis 12 (3): 132-140 (2019)}

Resumo. No presente estudo avaliou-se o efeito de plantas herbáceas floríferas (PHF) e plantas espontâneas (PE) sobre Dermaptera em algodoeiro colorido. O experimento foi conduzido em área com algodoeiro, Gossypium hirsutum L., cultivar BRS verde (Malvaceae), contendo bordas com plantas herbáceas floríferas (PHF) e plantas espontâneas (PE), durante o período de outubro/2011 a maio/2013. A amostragem foi quinzenal utilizando-se armadilhas tipo alçapão (Pitfall). Os dados obtidos foram submetidos às análises de fauna e variância. O total de 3.527 indivíduos e quatro espécies de Dermaptera foram capturados no algodoeiro colorido e plantas herbáceas. As espécies de dermápteros predominante foi Labidura riparia (Pallas). A maior diversidade de espécies de dermápteros ocorreu em plantas espontâneas $\left(H^{\prime}=0,661\right)$ e o maior número de indivíduos de em Fagopyrum esculentum Moench e plantas espontâneas.

Palavras-Chave: Análise faunística; Gossypium hirsutum; fatores meteorológicos; inimigos naturais.

\section{Conservative biological control: Herbaceous plants on diversity and abundance of dermaptera in colored cotton}

\begin{abstract}
The aim of this study was to evaluate the effect of flowering herbaceous plants (FHP) and weed plants (WP) on Dermaptera in colored cotton. The study was conducted in colored cotton, Gossypium hirsutum L., BRS Verde, with FHP and WP, from October/2011 to May/2013. The sampling was fortnightly with pitfall traps. The data were submitted to faunal analysis and analysis of variance (ANOVA). The total of 3,527 individuals and four species of Dermaptera were captured in colored cotton and herbaceous plants. Labidura riparia (Pallas) were Dermaptera predominant, respectively. The higher diversity of species for Dermaptera the greater number was in Fagopyrum esculentum Moench and weed plants $\left(H^{\prime}=0,661\right)$.
\end{abstract}

Keywords: Faunistic analysis; Gossypium hirsutum; natural enemies; weather factors.

(0) controle biológico conservativo é uma técnica que visa conservar e aumentar o número de inimigos naturais nos agroecossistemas (Thomas et al. 1991; PfiffNer \& WYss 2004). Essa técnica pode ser incorporada a programas de manejo integrado de pragas (Coluins et al. 2003 a, b) e é importante para a sustentabilidade (ALTIERI et al. 2003) e a biodiversidade na agricultura (BENGTSSON et al. 2005; Hole et al. 2005). Nesse sentido, alguns autores (THOMAs et al. 1992; ALTIERI 2004; BROWN et al. 2010) sugeriram a criação de faixas de plantas herbáceas nas adjacências de cultivos agrícolas para proporcionarem áreas de refúgio e proteção para artrópodes predadores, contribuindo para a rápida colonização das culturas por esses organismos.

Entretanto, o algodoeiro pode ser infestado por inúmeras pragas, que podem causar até 30\% de quebra na produção (MichelotTo et al. 2013). Quanto aos inimigos naturais associados ao algodoeiro e áreas de refúgio destacam-se os insetos das famílias Labiduridae, Forficulidae e Anisolabididae (Dermaptera) como importantes agentes de controle biológico de insetos-pragas (Majerus 1994; Gondim et al. 2001; Hannam; et al. 2008; ANDREASSEN et al. 2009).

Os dermápteros são predadores de ovos e formas jovens de Lepidoptera, Hemiptera, Coleoptera e Diptera (Costa et al. 2007; Silva et al. 2010 a, b). Para aumentar a efetividade como agentes de controle biológico de pragas, há necessidade de se avaliar a influência das culturas e dos diferentes tipos de habitat presentes nos agroecossistemas para a identificação de componentes que proporcionem as melhores condições para esses predadores atuarem sobre as pragas (LÖVEI \& SUNDERLAND 1996; Holland \& LUFF 2000). Informações disponíveis sobre esse assunto já demonstraram os benefícios do controle natural de pragas em agroecossistemas de clima temperado (Collins et al. 2003b; FIEDLER \& LANDIS 2007).

O aproveitamento desses insetos predadores no controle de pragas está vinculado às práticas como a introdução de cercas
Edited by:

William Costa Rodrigues

\section{Article History:}

Received: 19.ii.2019

Accepted: 02.x.2019
Corresponding author:

João Marcos Amario Sousa

७ agroamario@outlook.com

(1D) No ORCID record
Funding agencies:

$\checkmark$ Without funding declared 
vivas e de faixas de gramíneas ou de plantas floríferas nos agroecossistemas (MACLEOD et al. 2004; WHITE et al. 2012). No Brasil existem poucos relatos da influência de plantas herbáceas sobre inimigos naturais em culturas agrícolas. As informações existentes relatam a utilização de vegetação de cobertura para aumentar o número de inimigos naturais em culturas perenes (Altieri et al. 2003; PeREs et al. 2009; White et al. 2012), além do plantio de vegetação nas bordas das culturas para atração de parasitoides de lepidópteros (PERES 2007; 2008; PFIFFNER et al. 2009) e outros inimigos naturais (DEMITE \& FEREZ 2005).

No presente estudo avaliou-se o efeito de plantas herbáceas floríferas (PHF) e plantas espontâneas (PE) sobre Dermaptera em algodoeiro colorido. Para isso procurou-se: (i) identificar a composição de espécies e determinar os índices de diversidade, equitabilidade e avaliar a similaridade das comunidades desses besouros predadores associados ao algodoeiro colorido adjacente a PHF e PE, (ii) estudar a flutuação populacional e verificar a influência dos fatores meteorológicos sobre as espécies predominantes, (iii) avaliar o efeito da presença de espécies de PHF e PE na ocorrência de dermápteros no algodoeiro.

\section{MATERIAL E MÉTODOS}

Descrição e implantação da área experimental. $O$ estudo foi conduzido durante o período de 2011 a 2013, em área experimental da Fazenda de Ensino, Pesquisa e Produção (FEPE) e no Laboratório de Ecologia de Insetos (LECOL) do Departamento de Fitossanidade, Faculdade de Ciências Agrárias e Veterinárias (FCAV), Universidade Estadual Paulista "Júlio de Mesquita Filho” (UNESP), Câmpus de Jaboticabal, SP. A área experimental apresenta solo tipo Latossolo Vermelho Distrófico e com as seguintes coordenadas geográficas: $21^{\circ} 15^{\prime} 22$ " $\mathrm{S}$, $48^{\circ} 18$ ' $58^{\prime \prime}$ W, altitude $595 \mathrm{~m}$, a temperatura anual média foi de $20,5^{\circ} \mathrm{C}$ e precipitação pluviométrica anual média de $1.425 \mathrm{~mm}$.
As sementes do algodoeiro colorido, Gossypium hirsutum L. latifolium Hutch., herbáceo, cultivar BRS verde foram obtidas junto à Embrapa Algodão, localizada em Campina Grande, PB. A referida cultivar foi selecionada para o estudo devido: (i) ser a mais indicada para o cultivo em localidades com precipitação pluvial igual ou acima de $600 \mathrm{~mm}$ anuais (CARvalHo 2005) e (ii) ser indicada para cultivo orgânico (HERCUlano et al. 2008). A semeadura foi realizada com o início das primeiras chuvas, compreendendo o período de outubro a novembro de 2011 e 2012. Porém, na entressafra promoveu-se a eliminação e destruição das plantas de algodoeiro de acordo a "Resolução SAA - 50, 14-10-2010" que determina o estabelecimento do vazio sanitário no período de 10 de julho a 10 de outubro para áreas cultivadas com algodoeiro no estado de São Paulo. Salienta-se que esse procedimento foi adotado somente para o algodoeiro na primeira safra (2011/2012) e na segunda safra (2012/2013), sendo mantidas as PHF e PE para a continuidade do experimento.

Foram implantadas cinco áreas (blocos) com algodoeiro colorido, cada uma com $40 \mathrm{~m}$ de comprimento por $40 \mathrm{~m}$ de largura, totalizando $8.000 \mathrm{~m}^{2}$ para cada período de condução experimental (Figura 1). O espaçamento adotado foi de $1 \mathrm{~m}$ entre linhas com plantas espaçadas de 0,3 a $0,5 \mathrm{~m}$, duas plantas por cova, e adubação com esterco bovino curtido incorporado ao solo na dose de $10 \mathrm{t} \mathrm{ha}^{-1}$. Ressalta-se que no desenvolvimento desse estudo não houve a utilização de produtos químicos e o plantio do algodoeiro foi feito manualmente.

Nas adjacências da área com algodoeiro colorido, havia a presença de dois fragmentos florestais (1,5 ha e 7,5 ha) e áreas de cultivo de soja ( $3 \mathrm{ha}$ ) e milho (o,5 ha), as quais, estavam presentes durante toda a execução do experimento (Figura 1).

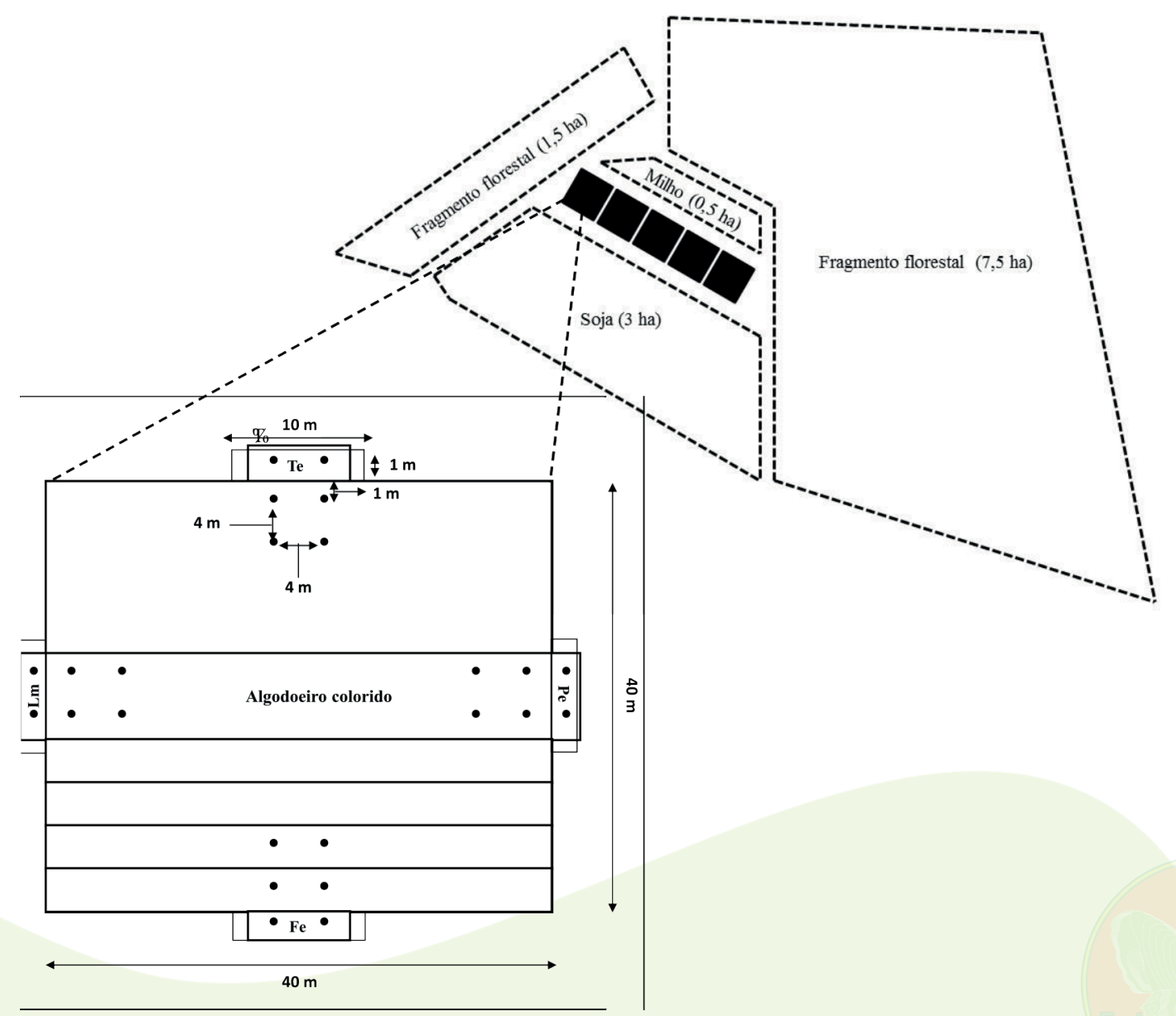

Figura 1. Representação esquemática indicando a localização dos cinco blocos com algodoeiro colorido e as bordas de plantas herbáceas, com destaque do bloco para análise ANOVA. O ponto negro $(\bullet)$ representa a posição das armadilhas tipo alçapão. Te - Tagetes erecta; Lm - Lobularia maritima; Fe - Fagopyrum esculentum; Pe - plantas espontâneas. 
Nas bordas da área com algodoeiro foram cultivadas plantas herbáceas mantidas em canteiros de $1 \mathrm{~m}$ x $10 \mathrm{~m}$ (Figura 1), sendo uma das bordas constituídas por plantas espontâneas (PE) e três bordas cultivadas com uma das seguintes espécies de plantas herbáceas floríferas (PHF): flor-de-mel [Lobularia maritima (L.) (Brassicaceae)], cravo-de-defunto [Tagetes erecta L. (Asteraceae)] e trigo sarraceno [Fagopyrum esculentum Moench (Polygonaceae)]. Essas espécies de PHF foram selecionadas por favorecerem a ocorrência de insetos predadores sem beneficiar insetos-praga (JohANowicz \& Mitchell 2000; SILVEIRA et al. 2009). A posição de cada borda com $P H F$ e $P E$ ao redor da área foi definida por sorteio, devido o delineamento experimental ter sido em blocos inteiramente casualizados, tendo como princípio o controle local, a distribuição ao acaso (BARBOSA \& MALDONADO JÚNIOR 2015).

Na borda com plantas espontâneas (PE) foram encontradas as seguintes espécies: Caruru - Amaranthus retroflexus L., Apaga-fogo - Alternanthera tenella Colla, Caruru-de-porco Amaranthus spinosus L. (Amaranthaceae); Guanxuma - Sida spinosa L. (Malvaceae), Capim-amargoso - Digitaria insularis (L.), Capim pé-de-galinha - Eleusine indica (L.) Gaer, Capim carrapicho - Cenchrus echinatus L. (Poaceae), Carrapichode-carneiro - Acanthospermum hispidum DC. (Asteraceae), Beldroega - Portulaca oleracea L. (Portulacaceae), Erva-botão Richardia brasiliensis Gomes (Rubiaceae), Leiteira - Euphorbia heterophylla L., Erva andorinha - Chamaesyce hyssopifolia (L.) Small (Euphorbiaceae), Trapoeraba - Commelina benghalensis L. (Commelinaceae), Anileira - Indigofera hirsuta L. (Fabaceae), Corda-de-viola - Ipomea grandifolia (Dammer) O’Donell (Convolvulaceae) (Figura 2A identificação dessas plantas foi feita pelo Dr. Vitor Fernandes Oliveira de Miranda, (FCAV/ UNESP), Câmpus de Jaboticabal, SP. e Dra. Núbia M. Correia da EMBRAPA Hortaliças, Brasília, DF.

O plantio de cada espécie de PHF foi feito por meio de mudas e sementes obtidas junto a empresas de jardinagem e floricultura de Jaboticabal, SP. Foram utilizados os espaçamentos entre mudas $(\ell)$ e densidade de plantas (D) recomendadas para cada espécie, conforme orientações do produtor, T. erecta $(\ell=15-30$ $\mathrm{cm} ; \mathrm{D}=200$ plantas) e as plantas apresentava altura de 40 a $60 \mathrm{~cm}, L$. maritima $(\ell=22-30 \mathrm{~cm} ; \mathrm{D}=149$ plantas $)$ e altura $(10$ a $20 \mathrm{~cm}), F$. esculentum $(\ell=5-10 \mathrm{~cm} ; \mathrm{D}=530$ plantas $)$ e altura (50 a $60 \mathrm{~cm})$. A adubação dos canteiros foi efetuada com a utilização de esterco bovino curtido incorporado ao solo na dose de $50 \mathrm{Kg}$ por canteiro. As espécies de PHF foram plantadas em cada canteiro três meses antes da semeadura do algodoeiro na primeira safra, compreendendo o período de agosto de 2011, para que à fase de florescimento das plantas coincidisse com a data de semeadura do algodoeiro e início das amostragens. Ressalta-se que para a segunda safra, as bordas com PHF permaneceram na área experimental, porém com manutenção periódica através do replantio de novas mudas em estágio fenológico de floração. A borda sem PHF foi permitido o crescimento de plantas espontâneas (Figura 1).

Amostragem e identificação dos insetos. A amostragem dos insetos foi realizada utilizando-se armadilhas tipo alçapão ("pitfall trap") constituídas de copos plástico de $8 \mathrm{~cm}$ de diâmetro e $14 \mathrm{~cm}$ de altura, contendo $100 \mathrm{~mL}$ de solução a base de água (97,2 mL); formaldeído P.A. 36,5 - 38,o \% (2,7 mL) e detergente neutro $(0,1 \mathrm{~mL})$. Todas as armadilhas foram cobertas com pratos plásticos de $20 \mathrm{~cm}$ de diâmetro, com altura suficiente para permitir a captura dos insetos e minimizar a entrada da água da chuva.

Ainstalação das armadilhas ocorreu nos canteiros eno algodoeiro, totalizando 24 armadilhas por bloco, sendo distribuídas igualmente em quatro dos cinco blocos (Figura 1). As armadilhas ficaram distantes $4 \mathrm{~m}$ entre si sendo distribuídas como segue: no centro de cada canteiro instalou-se duas armadilhas, enquanto no interior do algodoeiro foram instaladas 4 armadilhas ficando duas armadilhas a $1 \mathrm{~m}$ e duas a $5 \mathrm{~m}$ da borda dos canteiros, de tal modo que a distância horizontal entre elas foi 4 m (Figura 1).

A amostragem dos dermápteros foi feita continuamente no período de março/2012 a maio/2013, totalizando 18 datas amostrais. Após instaladas, as armadilhas permaneceram no campo por 15 dias sendo, após este período, substituídas por novas armadilhas. Os insetos capturados foram mantidos em recipientes de $50 \mathrm{~mL}$ contendo álcool $70 \%$ para posterior triagem. Na triagem utiliza-se bandeja de $40 \mathrm{~cm}$ x $20 \mathrm{~cm}$, peneira de nylon $12 \mathrm{~cm}$, pincel e pinça para separar os insetos dos demais materiais como: solo, restos vegetais e outros invertebrados e vertebrados.

Para a identificação das espécies, inicialmente o material coletado foi separado em morfoespécies com auxilio da coleção existente no Laboratório de Ecologia de Insetos (LECOL), Departamento de Fitossanidade, FCAV/UNESP. Após esta etapa, as espécies de Dermaptera foram identificadas pelo Dr. Javier Layme Manchego, Servicio Nacional de Sanidad Agraria, Lima, Peru. Os exemplares identificados foram depositados na coleção de insetos do LECOL.

\section{Análise dos dados}

Análise de fauna e flutuação populacional. A análise de fauna dos dermápteros proporcionou determinar as espécies predominantes, as quais foram caracterizadas por alcançarem as categorias máximas de dominância, abundância, frequência e constância (Silveira Neto et al. 1976; 1995; 2014). Pela análise também foram obtidos os índices de diversidade de Shannon-Wiener (H') e equitabilidade (E) para os tratamentos (PHF e PE) e algodoeiro. A variância de H' permitiu calcular o intervalo de confiança de 95\% (IC) desse índice. O intervalo de confiança calculado permitiu determinar a diferença estatística entre os índices de diversidade das espécies de insetos. Essas informações foram obtidas utilizando-se o software ANAFAU (MORAEs et al. 2003).

Para calcular o índice de similaridade de Sorensen $\left(\mathrm{SO}_{\mathrm{ij}}\right)$ foi comparado o total de indivíduos capturados em cada tratamento (PHF e PE) e algodoeiro, também obtido pelo software ANAFAU.

A flutuação populacional foi feita para todas as espécies de dermápteros encontradas. As figuras foram obtidas plotandose o total de espécimes por data de amostragem ao longo de 18 coletas, apresentando um intervalo entre as safras de 2012 e 2013 denominado de entressafra. Ressalta-se que em cada data de amostragem foi identificado o período fenológico do algodoeiro, conforme metodologia de MARUR \& RUANO (2003).

Influência de fatores meteorológicos. A influência de fatores meteorológicos foi avaliada para as espécies de dermápteros, devido o elevado número de indivíduos e o baixo número de espécies observadas. Utilizou-se análise de regressão múltipla pelo método stepwise, no qual se considerou o nível de 10\% de significância para a inclusão de variável independente. Os fatores meteorológicos considerados foram: temperaturas máxima, mínima e média $\left({ }^{\circ} \mathrm{C}\right)$, precipitação pluvial $(\mathrm{mm})$, umidade relativa (\%) e radiação solar, registrados pela Estação Agroclimatológica da FCAV/UNESP. Para os fatores meteorológicos temperatura, umidade relativa e radiação solar foram representados pela média e precipitação pluvial pela soma dos valores desses fatores registrados nos quinze dias que antecederam a cada data de amostragem das espécies. As análises foram realizadas no software SAS institute, versão 9.o.

Efeito de plantas herbáceas. Para avaliar o efeito de PHF e PE sobre a densidade relativa das espécies de dermápteros foi utilizado o delineamento em blocos casualizados com parcelas 
subdivididas (Split-plot), com quatro tratamentos (bordas com PHF e PE) (Figura 1) e cinco repetições. Os dados foram transformados em $\log (\mathrm{x}+5)$ para normalidade e estabilidade da variância e submetidos à análise de variância. As médias foram comparadas pelo teste de Tukey, a $5 \%$ de probabilidade. A análise foi efetuada por meio do software AgroEstat Versão 1.1.0.711 (BARbosa \& MALDONADO JÚNIOR 2015). Nesta análise foram consideradas as espécies com número acima de 10 indivíduos coletados durante o estudo.

\section{RESULTADOS E DISCUSSÃO}

Análise de fauna e flutuação populacional. $O$ total de 3.527 indivíduos de dermápteros pertencentes a quatro espécies, quatro gêneros e três famílias foram coletados na área cultivada com algodoeiro colorido e plantas herbáceas (Tabela 1). Essas espécies já foram registradas ocorrendo na região de Jaboticabal, SP (MARTINS 2011; RAMOS 2015; OTUKA 2015).

A única espécie classificada como predominante foi Labidura riparia (Pallas) (Dermaptera: Labiduridae), que apresentou elevada abundância (3.010 indivíduos), sendo responsável por 85,34\% do total de indivíduos capturados, resultado semelhante ao obtido por RAmos (2015) em consórcio de couve e sorgo, em que $L$. riparia representou $98,65 \%$ do total de dermápteros capturados. Em áreas degradas e de pastagem L. riparia também foi considerada predominante (LiU et al. 2014). Essa espécie de tesourinha já foi observada predando ovos e lagartas de lepidópteros em milho, algodão e repolho (SHEPARD et al. 1973; AMMAR \& FARRAG 1974; STRANDBERG 1981; HAY-RoE et al. 2016), e Drosophila suzukii (Matsumura) (Diptera: Drosophilidae) em pomares de diversas frutíferas (GABARRA et al. 2015). Labidura riparia foi o principal predador da lagarta-do-cartucho,

Tabela 1. Resultado da análise de fauna das espécies de Dermaptera. Jaboticabal, SP. 2012/2013.

\begin{tabular}{lcc}
\hline Espécies & No de Indivíduos & $\%$ \\
\hline${ }^{1}$ Labidura riparia (Pallas) & 3010 & 85,34 \\
\hline${ }^{2}$ Euborellia sp. & 336 & 9,53 \\
\hline${ }^{3}$ Doru luteipes (Scudder) & 174 & 4,93 \\
\hline${ }^{3}$ Kleter sp. & 7 & 0,20 \\
\hline Total de espécies & $\mathbf{4}$ & \\
Total de Indivíduos & $\mathbf{3 5 2 7}$ & \\
H' \pm IC & $\mathbf{0 , 5 2 0} \pm \mathbf{0 , 0 1}$ & $\mathbf{0 , 3 7 5}$ \\
$\mathbf{E}$ & & \\
\hline
\end{tabular}

Espécies predominantes em negrito

Famílias: ${ }^{1}$ Labiduridae; ${ }^{2}$ Anisolabididae; ${ }^{3}$ Forficulidae.

H' = Índice de Diversidade de Shannon-Wiener, IC = intervalo de confiança a 95\%, E = Índice de Equitabilidade, (\%) = porcentagem do número total de cada espécie.

Tabela 2. Índice de diversidade de Shannon-Wiener (H’) e equitabilidade (E) de Dermaptera em vários habitats. Jaboticabal, SP. $2012 / 2013$.

\begin{tabular}{|c|c|c|c|c|c|}
\hline \multirow{2}{*}{ Índices } & \multicolumn{5}{|c|}{ Habitat } \\
\hline & Algodão & Fe & Lm & $\mathbf{P e}$ & Te \\
\hline H' & $\mathrm{o}, 471 \mathrm{c}$ & $0,613 \mathrm{~b}$ & $\mathrm{o}, 424 \mathrm{e}$ & $0,661 \mathrm{a}$ & $0,447 \mathrm{~d}$ \\
\hline $\mathbf{E}$ & 0,428 & 0,558 & 0,306 & 0,477 & 0,407 \\
\hline
\end{tabular}

$\mathrm{Fe}=$ Fagopyrum esculentum, $\mathrm{Lm}=$ Lobularia maritima, $\mathrm{Pe}=$ Plantas espontâneas, $\mathrm{Te}=$ Tagetes erecta .

Valores seguidos pela mesma letra minúscula na horizontal, não diferem entre si, através da sobreposição do intervalo de confiança.

O quociente de similaridade entre as comunidades de dermápteros presentes em F. esculentum - T. erecta e L. marítima - plantas espontâneas foi $100 \%$ (Tabela 3 ). Tal fato pode indicar que plantação de milho na Flórida (HAY-RoE et al. 2016), esta espécie de Spodoptera também é considerada praga da cultura do algodão 2010). Possivelmente, a capacidade predatória de $L$. riparia e sua dispersão devido a colheita das culturas de milho e soja próximas da área experimental explicam o número elevado

Por outro lado, Doru luteipes (Scudder) e Kleter sp. (Dermaptera: Forficulidae) foram as espécies com o menor número de ivíduos capturados, correspondendo juntas a 5,13\% do total (Tabela 2). Este resultado difere do observado por MARTINS (2011) que relatou estas espécies como as mais abundantes na cultura da soja.

Os índices de diversidade de Shannon-Winner e equitabilidade obtidos para o total de dermápteros capturados foram 0,520 e 0,375 , respectivamente (Tabela 1 )

A elevada diversidade de espécies de dermápteros nas bordas com plantas espontâneas pode estar relacionada com a diversidade de espécies vegetais encontradas nesse habitat, pois segundo HeRnÁNDEZ-RUIZ \& CASTAÑo-MENESES (2006) habitat heterogêneos são apontados como fator principal para a ocorrência de elevada diversidade de espécies de insetos dores, devido apresentarem mais recursos para nidificação e forrageamento, além de proporcionarem sombreamento, umidade e serrapilheira para espécies que habitam o solo.

Considerando as plantas herbáceas e o algodoeiro separadamente, as plantas espontâneas apresentaram o maior índice de diversidade $\left(\mathrm{H}^{\prime}=0,661\right)$, diferindo significativamente dos demais, enquanto $F$. esculentum apresentou elevado índice de equitabilidade (Tabela 2). 
Tabela 3. Resultado do Quociente de Similaridade de Sorensen $\left(\mathrm{SO}_{\mathrm{ij}}\right)$ para as espécies de dermápteros associados as diferentes plantas herbáceas e algodoeiro. Jaboticabal, SP. 2012/2013.

\begin{tabular}{rcccc}
\hline & Fagopyrum esculentum & Lobularia maritima & Plantas espontâneas & Tagetes erecta \\
\hline Algodão & $100,00 \mathrm{a}$ & $85,71 \mathrm{~b}$ & $85,71 \mathrm{~b}$ & $100,00 \mathrm{a}$ \\
Fagopyrum esculentum & - & $85,71 \mathrm{~b}$ & $85,71 \mathrm{~b}$ & $100,00 \mathrm{a}$ \\
Lobularia maritima & - & - & $100,00 \mathrm{a}$ & $85,71 \mathrm{~b}$ \\
Plantas espontâneas & - & - & - & $85,71 \mathrm{~b}$ \\
\hline
\end{tabular}

Valores de $\mathrm{SO}_{\mathrm{ij}}$ seguidos da mesma letra não se diferem entre si, através da sobreposição do intervalo de confiança.

Em relação à flutuação populacional, L. riparia e Euborellia sp. ocorreram em todas as datas de amostragem e a abundância discrepante observada entre essas duas espécies de tesourinhas pode estar associada à competição interespecífica, além do comportamento agressivo e ocorrência de canibalismo entre indivíduos desses predadores (DOBLER \& KõLLIKER 2010).

Os maiores picos populacionais de Labidura riparia ocorreram na primeira safra em 03/04/12 e na segunda safra em 21/02/2013, 05/03/2013, 20/03/2013 e 16/04/2013. Essas datas coincidem com o estádio reprodutivo do algodoeiro (Figura 1). Considerado o estádio de maior incidência de insetos-praga como pulgões e lagartas de Lepidoptera (MARUR \& RUANO 2003). Possivelmente, a presença de $L$. riparia pode estar associada com sua eficiência no controle de ovos e formas jovens de Lepidoptera (HAY-RoE et

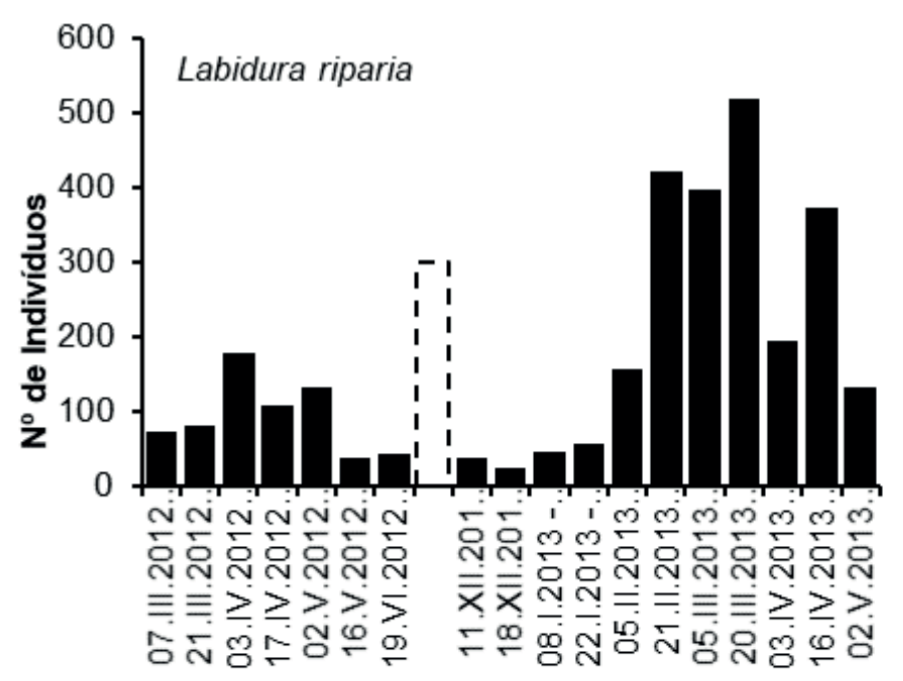

al. 2016). Por outro lado, GALli et al. (2003) verificaram que a maior ocorrência de espécies de Labiduridae em pomar de goiaba foi entre os meses de janeiro a março, semelhante ao período observado nesse estudo.

Na safra de 2011/2012, os picos populacionais de Euborellia sp. ocorreram no estádio reprodutivo de desenvolvimento do algodoeiro, 16/05/12 e 19/06/12, na safra 2012/2013, os picos foram observados em 11/12/2012 e 20/03/2013, quando o algodoeiro apresentava os estádios vegetativo e reprodutivo, respectivamente (Figura 2). Vários autores (RAMALHO \& WANDERLEY 1996; Lemos et al. 1998; Silva et al. 2010 a, b) ressaltam Euborellia annulipes (Lucas) (Dermaptera: Anisolabididae) como importante inimigo natural de larvas e pupas de Anthonomus grandis (Boheman) (Coleoptera:
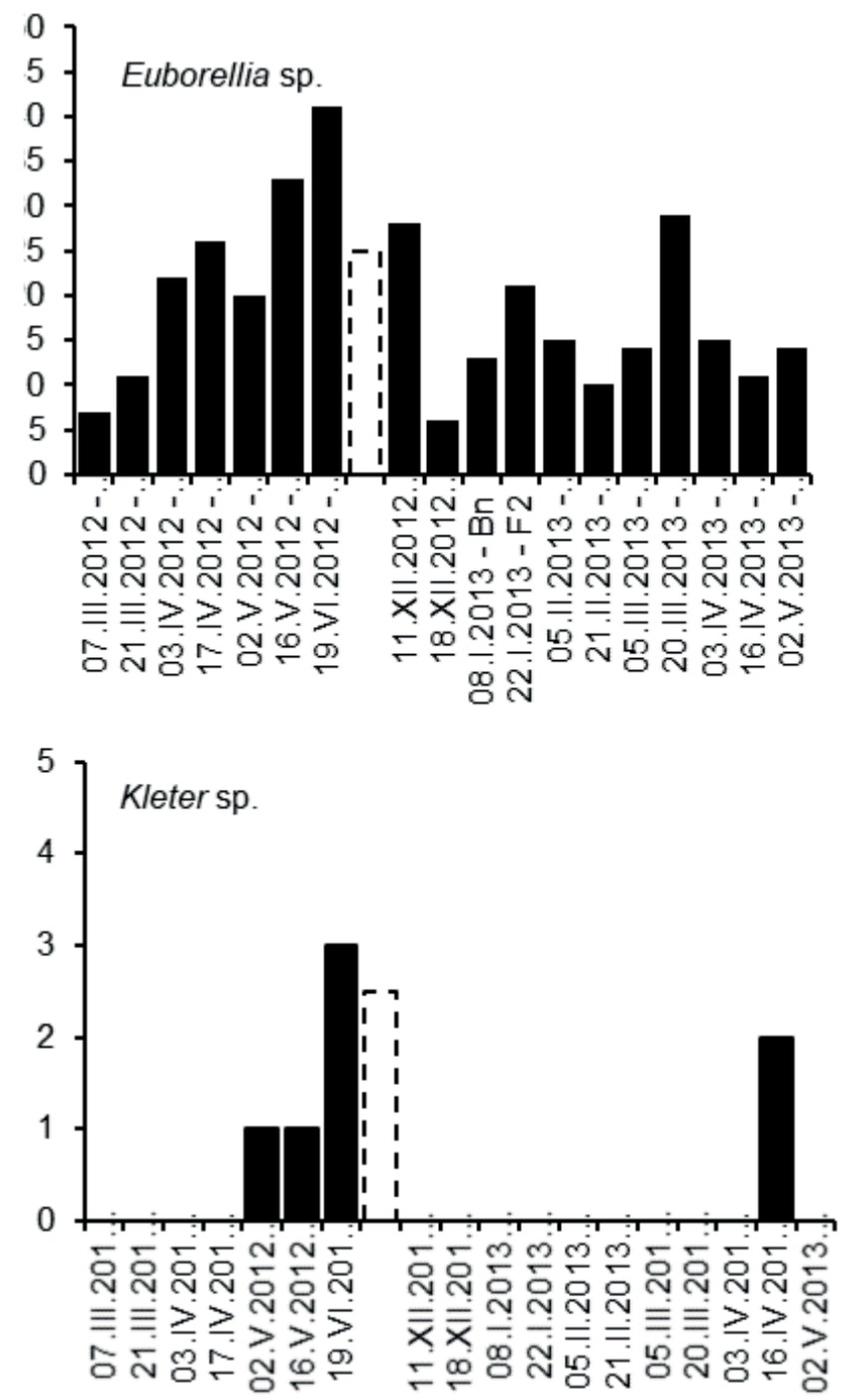

\section{Fenologia / Datas de amostragem}

Figura 2. Flutuação populacional das espécies de Dermaptera. Jaboticabal, SP. 2012/2013. Linhas tracejadas representam o período de entressafra entre os meses 07 a 11/2012. Fenologia do algodoeiro: Período vegetativo (V), período reprodutivo $(\mathrm{B}=$ botão, $\mathrm{F}=$ flor, $\mathrm{C}=\mathrm{capulho}$ ). 
Curculionidae), e os pulgões Hyadaphis foeniculi (Passerini) e Brevicoryne brassicae (Linnaeus) (Hemiptera: Aphididade).

A espécie $D$. luteipes não ocorreu nas duas primeiras datas de amostragem das duas safras avaliadas. Na segunda safra esta espécie foi mais numerosa que na primeira, ocorrendo picos populacionais em 05/03/2013 e 20/03/2013, quando o algodoeiro estava no período reprodutivo. Kleter sp. apresentou picos populacionais em 02/05/2012, 16/05/2012 e 19/06/2012 na primeira safra e somente em 16/05/2013 na segunda safra com baixo número de indivíduos.

Influência de fatores meteorológicos. Nenhuma variável meteorológica atingiu o nível mínimo de significância para entrar no modelo multivariado para os dermápteros $L$. riparia e D. luteipes (Tabela 4), ou seja, não influenciou neste estudo em que os fatores meteorológicos foram: Temperatura (Tmín = 14,41 ${ }^{\circ} \mathrm{C}$ e Tmáx $=33,44{ }^{\circ} \mathrm{C}$ ), Precipitação pluvial (o a 297,60 mm), Umidade relativa $(29,30$ a $105,59 \%)$ e Radiação solar $(12,30$ a 23,99 $\mathrm{MJ} \mathrm{m}^{-2}$ ). GuERREIRo et al. (2003) também não obtiveram correlação com fatores meteorológicos para D. luteipes em milho.
Para Euborellia sp. o modelo selecionou uma única variável significativa e negativa que foi a temperatura mínima, explicando $18,09 \%$ da variação numérica da espécie (Tabela 4). Tal resultado sugere que com o incremento deste fator ocorre redução da densidade populacional da espécie. MARTins (2011) e OTUKA (2015) obtiveram resultados diferentes do observado nesse estudo, onde a temperatura mínima influiu de forma positiva para Euborellia sp.

O modelo multivariado obtido para Kleter sp. selecionou apenas a radiação solar como variável significativa e negativa, podendo indicar uma diminuição desse predador com o aumento desse fator abiótico (Tabela 4).

Efeito de plantas herbáceas. Para as diferentes espécies de plantas herbáceas consideradas, verificou-se efeito significativo somente sobre o número médio de indivíduos de $L$. riparia em F. esculentum (Tabela 5). Considerando o número médio de indivíduos relacionados às plantas herbáceas, verificou que para as espécies de dermápteros encontrados variou de 0,70 a 1,49 e para o algodão variou de 0,70 a 1,89 . Plantas de $F$. esculentum são predispostas ao acamamento, o que pode ter favorecido o forrageamento e fornecido proteção para às fases jovens e adulta dessa espécie de dermaptera (CRUZ et al. 1995).

Tabela 4. Resultado de modelos ajustados pelo método stepwise entre as espécies de Dermaptera e fatores meteorológicos. Jaboticabal, SP. $2012 / 2013$.

\begin{tabular}{lcccc}
\hline \multicolumn{1}{c}{ Fatores abióticos } & \multicolumn{4}{c}{ Espécies } \\
\cline { 2 - 5 } & Labidura riparia & Euborellia sp. & Doru luteipes & Kleter sp. \\
\hline Intercepto & 167,222 & 56,139 & 9,666 & 4,081 \\
Temperatura máxima $\left({ }^{\circ} \mathrm{C}\right)$ & - & - & - & - \\
Temperatura mínima $\left({ }^{\circ} \mathrm{C}\right)$ & - & $-2,010$ & - & - \\
Temperatura média $\left({ }^{\circ} \mathrm{C}\right)$ & - & - & - & - \\
Precipitação pluvial $(\mathrm{mm})$ & - & - & - & - \\
Umidade relativa $(\%)$ & - & - & - & - \\
Radiação solar $\left(\mathrm{MJ} \mathrm{m}^{-2}\right)$ & - & - & - & $-0,203$ \\
$\mathbf{R}^{\mathbf{2}}(\mathbf{m o d e l o})$ & - & $\mathbf{0 , 1 8 0 9}$ & - & $\mathbf{0 , 5 8 2 8}$ \\
F & - & $\mathbf{3 , 5 3}$ & - & $\mathbf{2 2 , 3 5 *}$ \\
\hline
\end{tabular}

**, * Significativo a 1\% e $5 \%$ de probabilidade, respectivamente; $\mathrm{R}^{2}$ = Coeficiente de determinação.

Tabela 5. Número médio ( \pm erro padrão) de Dermaptera em algodoeiro colorido e plantas herbáceas. Jaboticabal, SP. $2012 / 2013$.

Plantas Herbáceas

\begin{tabular}{llllll} 
Espécies/Habitat & \multicolumn{4}{c}{ Plantas Herbáceas } & CV \\
\cline { 2 - 5 } & $\begin{array}{l}\text { Fagopyrum } \\
\text { esculentum }\end{array}$ & $\begin{array}{l}\text { Lobularia } \\
\text { marítima }\end{array}$ & $\begin{array}{c}\text { Plantas } \\
\text { espontâneas }\end{array}$ & Tagetes erecta & F \\
\hline
\end{tabular}

\section{Doru luteipes}

$\begin{array}{rllllll}\text { PHF + PE } & 0,89 \pm 0,07 \mathrm{Aa} & 0,76 \pm 0,03 \mathrm{Aa} & 0,84 \pm 0,08 \mathrm{Aa} & 0,79 \pm 0,08 \mathrm{Aa} & 0,68 \mathrm{~ns} & 18,42 \\ \text { Algodão } & 0,97 \pm 0,06 \mathrm{Aa} & 0,94 \pm 0,05 \mathrm{Aa} & 0,97 \pm 0,04 \mathrm{Aa} & 0,86 \pm 0,06 \mathrm{Aa} & 0,63 \mathrm{~ns} & 15,81\end{array}$

Euborellia sp.

$\begin{array}{rrrrrrr}\text { PHF + PE } & 1,03 \pm 0,06 \mathrm{Aa} & 0,99 \pm 0,02 \mathrm{Aa} & 1,08 \pm 0,12 \mathrm{Aa} & 0,86 \pm 0,09 \mathrm{Aa} & 1,23 \mathrm{~ns} & 21,76 \\ \text { Algodão } & 1,06 \pm 0,12 \mathrm{Aa} & 0,95 \pm 0,04 \mathrm{Aa} & 1,07 \pm 0,03 \mathrm{Aa} & 0,90 \pm 0,06 \mathrm{Aa} & 0,94 \mathrm{~ns} & 15,82\end{array}$

Kleter sp.

$\begin{array}{rrrrrrr}\text { PHF + PE } & 0,70 \pm 0,00 A a & 0,70 \pm 0,00 A a & 0,77 \pm 0,06 \mathrm{Aa} & 0,70 \pm 0,00 \mathrm{Aa} & 1,86 \mathrm{~ns} & 7,97 \\ \text { Algodão } & 0,70 \pm 0,00 \mathrm{Aa} & 0,71 \pm 0,01 \mathrm{Aa} & 0,70 \pm 0,00 \mathrm{Aa} & 0,70 \pm 0,00 \mathrm{Aa} & 0,10 \mathrm{Ons} & 7,83 \\ \text { riparia } & & & & & \\ \text { PHF + PE } & 1,49 \pm 0,08 \mathrm{Ba} & 1,21 \pm 0,19 \mathrm{Bab} & 1,02 \pm 0,08 \mathrm{Bab} & 1,34 \pm 0,11 \mathrm{Bb} & 3,27^{*} & 17,51 \\ \text { Algodão } & 1,83 \pm 0,11 \mathrm{Aa} & 1,87 \pm 0,05 \mathrm{Aa} & 1,89 \pm 0,05 \mathrm{Aa} & 1,79 \pm 0,07 \mathrm{Aa} & 0,18 \mathrm{~ns} & 13,80\end{array}$

PHF = plantas herbáceas floríferas, $\mathrm{PE}=$ plantas espontâneas.

${ }^{1}$ Médias \pm erro padrão seguidas pela mesma letra maiúscula na vertical e minúscula na horizontal não diferem entre si, pelo teste de Tukey a $5 \%$ de probabilidade.

ns $=$ não significativo, ${ }^{*}$ significativo a $5 \%$ de probabilidade pelo teste $\mathrm{F}$. 
Alguns autores (LAVANDERO et al. 2005; WACKERS et al. 2006; WACKERS \& VAN RIJN 2012), descreveram que criação de refúgios em áreas agrícolas pode favorecer a ocorrência e distribuição de insetos predadores como as tesourinhas, pelo fornecimento de fontes alimentares alternativas como néctar e pólen. Ressaltase que esses recursos alimentares, complementa a dieta desses inimigos naturais, melhorando seus parâmetros biológicos como longevidade e fertilidade (RAHAT et al. 2005; Rose et al. 2006; VENZON et al. 2006).

Quando os habitats (plantas herbáceas e algodoeiro) foram comparados entre si, L. riparia foi a única espécie que apresentou diferença significativa no número médio de indivíduos associados a todas as plantas herbáceas e algodoeiro, sendo maior numericamente no algodoeiro (Tabela 5). Resultado que pode estar associado à eficiência desse predador no controle de insetos-praga presentes na cultura de algodão (HAY-RoE et al. 2016).

As espécies predominantes foram $L$. riparia (Dermaptera: Labiduridae), em que $F$. esculentum observou a maior diversidade de espécimes. As espécies $L$. riparia e $D$. luteipes não são influenciadas pelos fatores meteorológicos. Os maiores picos populacionais de $L$. ripariam ocorrem nos meses de fevereiro, março e abril, enquanto Euborellia sp. nos meses de maio e junho.

\section{REFERÊNCIAS}

Altieri, MA, NE Silva \& CI Nicholls, 2003. O papel da biodiversidade no manejo de pragas. Ribeirão Preto: Editora Holos, $226 \mathrm{p}$.

Altieri, MA, 2004. Agroecologia: a dinâmica produtiva da agricultura sustentável. 4. ed. Porto Alegre: Editora da UFRGS. $120 \mathrm{p}$.

Ammar, ED \& SM Farrag, 1974. Studies on the behavior and biology of the earwig L. riparia Pallas (Dermaptera, Labiduridae). Journal of Applied Entomology, 75: 189-196. DOI: https://doi.org/10.1111/j.1439-0418.1974.tb01843.x.

Andreassen, LD, U Kuhlmann, PG Mason \& NJ Holliday, 2009. Host range testing of a prospective classical biological control agent against cabbage maggot, Delia radicum, in Canada. Biological Control, 48: 210-220, 2009. DOI: https://doi.org/10.1016/i.biocontrol.2008.10.006.

Barbosa, JC \& W Maldonado Júnior, 2015. AgroEstat - Sistema para Análises Estatísticas de Ensaios Agronômicos. Versão 1.1.0.711. Jaboticabal: Unesp.

Bengtsson, J, J Ahnström \& A Weibull, 2005. The effects of organic agriculture on biodiversity and abundance: a metaanalysis. Journal of Applied Ecology, 42: 261-269.

Brown, MW, CR Mathews \& G Krawczyk, 2010. Extrafloral nectar in an apple ecosystem to enhance biological control. Journal of Economic Entomology, 103: 1657-1664. DOI: https://doi.org/10.1603/EC10019.

Carvalho, LP, 2005. Algodão de fibra colorida no Brasil. In: Anais do $5^{\circ}$ Congresso Brasileiro De Algodão, Salvador. p. 1-3. Available on: <http://www.cnpa.embrapa.br/produtos/ algodao/publicacoes/trabalhos cba5/291.pdf $>$. [Acessed in: 30.ix.2015].

Collins, KL, ND Boatman, A Wilcox \& JM Holland, 2003a. A 5 -year comparison of overwintering polyphagous predator densities within a beetle bank and two conventional hedgebanks. Annals of Applied Biology, 143: 63-71. DOI: https://doi.org/10.1111/j.1744-7348.2003.00063.x.

Collins, KL, ND Boatman, A Wilcox \& JM Holland, 2003b. Effects of different grass treatments used to create overwintering habitat for predatory arthropods on arable farmland. Agriculture, Ecosystems and Environment, 96: 59-67. DOI: https://doi.org/10.1016/s0167-8809(03)00032-x.

Costa, NP, HD Oliveira, CH Brito \& AB Silva, 2007. Influência do nim na biologia do predador Euborellia annulipes e estudos de parâmetros para sua criação massal. Revista de Biologia e Ciências da Terra, 7: 1-8.

Cruz, I, CD Alvarenga \& PEF Figueiredo, 1995. Biologia de Doru luteipes (Scudder) e sua capacidade predatória de ovos de Helicoverpa zea (Boddie). Anais da Sociedade Entomologica do Brasil, 24: 273-278.

Demite, PR \& RJ Feres, 2005. Influência da vegetação vizinha na distribuição de ácaros em seringal (Hevea brasilliensis Muell. Arg. Euphorbiaceae) em São José do Rio Preto, SP. Neotropical Entomology, 34: 829-883. DOI: https://doi. org/10.1590/s1519-566x2005000500016.

Dobler, R \& M Kölliker, 2010. Kin-selected siblicide and cannibalism in the European earwig. Behavioral Ecology, 21: 257-263, DOI: https://doi.org/10.1093/beheco/arp184.

Fiedler, AK \& DA Landis, 2007. Attractiveness of Michigan native plants to Arthropod natural enemies and herbivores. Environmental Entomology, 36: 751-765.

Gabarra, R, J Riudavets, GA Rodríguez, J Pujade-Villar \& J Arnó, 2015 Prospects for the biological control of Drosophila suzukii. BioControl, 60: 331-339. DOI https://doi.org/10.1007/ s10526-014-9646-z.

Galli, JC, KCA Senô \& FJ Cividanes, 2003. Flutuação populacional de Labidura sp. em Psidium guajava submetido a dois métodos de pulverização de fenthion. Manejo Integrado de Plagas y Agroecología, 69: 45-49.

Gondim, DMC, JL Belot, P Silvie, \& N Petit, 2001. Manual de identificação das pragas, doenças, deficiências minerais e injúrias do algodoeiro do Brasil. 3. Ed. Cascavel: Codetec/ CIRAD (Boletim técnico, 33), 120 p.

Guerreiro, JC, EB Filho \& AC Busoli, 2003. Ocorrência estacional de Doru luteipes na cultura do milho em São Paulo, Brasil. Manejo Integrado de Plagas y Agroecología, 70: 46-49.

Hannam, JJ, JK Liebherr \& AE Hajek, 2008. Climbing behaviour and aphid predation by Agonum muelleri (Coleoptera: Carabidae). The Canadian Entomologist, 140: 203-207. DOI: https://doi.org/10.4039/no7-056.

Hay-Roe, MM, RL Meagher, RN Nagoshi \& Y Newman, 2016. Distributional patterns of fall armyworm parasitoids in a corn field and a pasture field in Florida. Biological Control, 96: 4856. DOI: https://doi.org/10.1016/j.biocontrol.2016.02.003.

Herculano, FC, WS Lira, GA Cândido \& ACF Vasconcelos, 2008. Índice de desenvolvimento sustentável no setor agrícola: um estudo de caso da tecnologia do algodão colorido algodão orgânico em Bom Sucesso - Paraíba. Engenharia Ambiental: Pesquisa e Tecnologia, 5: 14-23.

Hernández-Ruiz, P \& G Castaño-Meneses, 2006. Ants (Hymenoptera: Formicidae) diversity in agricultural ecosystems at Mezquital Valley, Hidalgo, Mexico. European Journal of Soil Biology, 42: 208-212. DOI: https://doi.org/10.1016/j.ejsobi.2006.07.020.

Hole, DG, AJ Perkins, JD Wilson, IH Alexander, F Grice \& $\mathrm{AD}$ Evans, 2005. Does organic farming benefit biodiversity? Biological Conservation, 122: 113-139. DOI: https://doi.org/10.1016/j.biocon.2004.07.018.

Holland, JM \& ML Luff, 2000. The effects of agricultural practices on Carabidae in temperate agroecosystems. Integrated Pest Management Reviews, 5: 109-129.

Johanowicz, DL \& ER Mitchell, 2000. Effects of sweet alyssum flowers on the longevity of the parasitoid wasps Cotesia marginiventris (Hymenoptera: Braconidae) and Diadegma insulare (Hymenoptera: Ichneumonidae). Florida Entomologist, 83: 41-47, 2000. DOI: https://doi.org/10.2307/3496226.

Kajak, A \& J Lukasiewicz, 1994. Do semi-natural patches enrich crop fields with predatory epigean arthropods? Agriculture, Ecosystems and Environment, 49: 149-161, 1994. DOI: https://doi.org/10.1016/0167-8809(94)90005-1.

Lavandero, B, SD Wratten, P Shishehbor \& S Worner, 2005. Enhancing the effectiveness of the parasitoid Diadegma semiclausum (Helen): Movement after use of 
nectar in the field. Biological Control, 34: 152-158, DOI: https://doi.org/10.1016/j.biocontrol.2005.04.013.

Lee, JC \& GE Heimpel, 2008. Effect of floral nectar, water, and feeding frequency of Cotesia glomerata longevity. Biocontrol, 53: 289-294, DOI: https://doi.org/10.1007/s10526-0079070-8.

Lemos, WP, RS Medeiros \& FS Ramalho, 1998. Influência da temperatura no desenvolvimento de Euborellia annulipes (Lucas) (Dermaptera: Anisolabididae), predador do Bicudo-do-Algodoeiro. Anais da Sociedade Entomológica do Brasil, 27: 67-76. DOI: https://doi.org/10.1590/s030180591998000100009 .

Liu, R, F Zhu, H AN \& Y Steinberger, 2014. Effect of naturally vs manually managed restoration on ground-dwelling arthropod communities in a desertified region. Ecological Engineering, 73: 545-552. DOI: https://doi.org/10.1016/j. ecoleng.2014.09.092.

Lövei, GL \& KD Sunderland, 1996. Ecology and behavior of ground beetles (Coleoptera: Carabidae). Annual Review of Entomology, Palo Alto, v. 41, p. 231- 256. DOI: https://doi.org/10.1146/annurev.en.41.010196.001311.

Macleod, A, SD Wratten, NW Sotherton \& MB Thomas, 2004. 'Beetle banks' as refuges for beneficial arthropods in farmland: long-term changes in predator communities and habitat. Agricultural and Forest Entomology, 6: 147-154. DOI: https://doi.org/10.1111/j.1461-9563.2004.00215.x.

Majerus, MEN, 1994. Ladybirds. London: HarperCollins, 367 p.

Martins, ICF, 2011. Insecta e arachnida associados ao solo: plantas herbáceas como área de refúgio visando ao controle biológico conservativo. Tese (Doutorado em Agronomia/Entomologia Agrícola) - Universidade Estadual Paulista "Júlio de Mesquita Filho”, Faculdade de Ciências Agrárias e Veterinária.

Marur, CJ \& O Ruano, 2003. Escala do Algodão. Londrina: IAPAR, 1-4 p. Informe da Pesquisa.

Miranda, JE, 2010. Manejo integrado de pragas do algodoeiro no cerrado brasileiro. Campina Grande: Embrapa: CNPA (Embrapa-CNPA. Circular Técnica, 131). 37 p.

Moraes, RCB, ML Haddad, S Silveira Neto \& AEL Reyes, 2003. Software para análise faunística - ANAFAU. Available on: $<$ http://www.lea.esalq.usp.br/softwares $>$.

Otuka, AK, 2015. Dinâmica populacional de insetos fitófagos e predadores em cana-de-açúcar sob aplicação de sílicio. Tese (Doutorado em Agronomia/Entomologia Agrícola) Universidade Estadual Paulista "Júlio de Mesquita Filho", Faculdade de Ciências Agrárias e Veterinária.

Peres, FSC, 2007. Cravo-de-defunto (Tagetes patula L.) como planta atrativa para tripés (Thysanoptera) e himenópteros parasitoide (Hymenoptera) em cultivo protegido. Dissertação (Mestrado em Agronomia/Entomologia Agrícola) - Faculdade de Ciências Agrárias e Veterinárias, Universidade Estadual Paulista.

Peres, FSC, OA Fernandes, LCP Silveira \& CSB Silva, 2009. Cravo-de-defunto como planta atrativa para tripes em cultivo protegido de melão orgânico. Bragantia, 68: 953-960. DOI: https://doi.org/10.1590/s0006-87052009000400015.

Pfiffner, L, H Luka, C Schlatter, A Juen, \& M Traugott, 2009. Impact of wildflower strips on biological control of cabbage lepidopterans. Agriculture, Ecosystems and Environment, 129: 310-314. DOI: https://doi.org/10.1016/j.agee.2008.10.003.

Pfiffner, L \& E Wyss, 2004. Use of wildflower strips to enhance natural enemies of agricultural pests, p. 165-186. In: GURR, G. M.; WRATTEN, S. D; ALTIERI, M. (Eds.). Ecological Engineering for Pest Management: Advances in Habitat Manipulation for Arthropods. CSIRO Publishing, 256 p. DOI: https://doi.org/10.1079/9780851999036.0165.

Rahat, S, GM Gurr, SD Wratten, JH MO \& R Neeson, 2005. Effect of plant nectar son adult longevity of the stink bug parasitoid, Trissolcus basalis. International Journal of Pest Management, 51: 321-324. DOI: https://doi.org/10.1080/09670870500312778.
Ramalho, FS \& PA Wanderley, 1996. Ecology and management of the boll weevil in South American cotton. American Entomologist, 42: 41-47. DOI: https://doi.org/10.1093/ ae/42.1.41.

Ramos, TO, 2015. Couve consorciada com sorgo e feijãoguandu na ocorrência de pulgões e insetos predadores. Tese (Doutorado em Agronomia/Entomologia Agrícola) Universidade Estadual Paulista "Júlio de Mesquita Filho", Faculdade de Ciências Agrárias e Veterinária.

Rose, USR, J Lewis \& JH Tumlinson, 2006. Extrafloral néctar from cotton (Gossypium hirsutum) as a food source for parasitic wasps. Functional Ecology, 20: 67-74. DOI: https://doi.org/10.1111/j.1365-2435.2006.01071.x.

Shepard, M, V Waddill \& W Kloft, 1973. Biology of the predaceous earwig Labidura riparia (Dermaptera: Labiduridae). Annals of Entomological Society of America, 66: 837-841, 1973. DOI: https://doi.org/10.1093/aesa/66.4.837.

Silva, AB, JL Batista \& CH Brito, 2010a. Aspectos biológicos de Euborellia annulipes (Dermaptera: Anisolabididae) alimentada com o pulgão Hyadaphis foeniculi (Hemiptera: Aphididae). Revista Caatinga, 23: 21-27.

Silva, AB, JL Batista \& CH Brito, 2010b. Capacidade predatória de Euborellia annulipes (Dermaptera: Anisolabididae) sobre Hyadaphis foeniculi (Hemiptera: Aphididae). Revista de Biologia e Ciências da Terra, 10: 44-51.

Silveira Neto, S, RC Monteiro, RA Zucchi \& RCB de Moraes, 1995. Uso da análise faunística de insetos na avaliação do impacto ambiental. Scientia Agricola, 52: 9-15. DOI: https://doi.org/10.1590/s0103-90161995000100003.

Silveira Neto, S, O Nakano, D Barbin \& NA Villa Nova, 1976. Manual de ecologia dos insetos. São Paulo: Agronômica Ceres, $419 \mathrm{p}$.

Silveira Neto, S, MLHaddad \& RCB Moraes, 2014. Artropodofauna aérea, p. 727-769. In: Berger, GU \& LRG Favoretto (Eds.) Monitoramento ambiental Soja Roundup Ready. Botucatu: FEPAF, Cap. 7.3.

Silveira, LCP, E Berti Filho, LSR Pierre, FSC Peres \& JNC Louzada, 2009. Marigold (Tagetes erecta L.) as an attractive crop to natural enemies in onion fields. Scientia Agricola, 66: 780-787. DOI: https://doi.org/10.1590/s0103-90162009000600009.

Strandberg, JO, 1981. Activity and abundance of the earwig, Labidura riparia, in a winter cabbage production ecosystem. Environmental Entomology, 10: 701-704. DOI: https://doi.org/10.1093/ee/10.5.701.

Thomas, MB, SD Wratten \& NW Sotherton, 1991. Creation of 'island' habitats in farmland to manipulate populations of beneficial arthropods: Predator densities and emigration. The Journal of Applied Ecology, 28: 906-917. DOI: https://doi.org/10.2307/2404216.

Thomas, MB, SD Wratten \& NW Sotherton, 1992. Creation of 'island' habitats in farmland to manipulate populations of beneficial arthropods: Predator densities and species composition. The Journal of Applied Ecology, 29: 524-531, DOI: https://doi.org/10.2307/2404521.

Venzon, M, MC Rosado, DE Euzébio, B Souza \& JH Schoereder, 2006. Suitability of leguminous cover crop pollens as food source for the green lacewing Chrysoperla externa (Hagen) (Neuroptera: Chrysopidae). Neotropical Entomology, 35: 371-376, DOI: https://doi.org/10.1590/s1519$566 \times 2006000300012$.

Wäckers, FL, JC Lee, GE Heimpel, K Winkler \& R Wagenaar, 2006. Hymenopteran parasitoids synthesize 'honeydewspecific' oligosaccharides. Functional Ecology, 20: 790-798. DOI: https://doi.org/10.1111/j.1365-2435.2006.01158.x.

Wäckers, FL \& PCJ Van Rijn, 2012. Pick and mix: selecting flowering plants to meet the requirements of target biological control insects, p. 139-165. In: Gurr, GM, SD Wratten, W Snyder \& DMY Read (Eds). Biodiversity and Insect Pests: Key Issues for Sustainable Management. Oxford: Wiley Blackwell. DOI: https://doi.org/10.1002/9781118231838.ch9. 
White, WH, TL Erwin \& BJ Viator, 2012. Leptotrachelus dorsalis

(Coleoptera: Carabidae): a candidate biological control agent

of the sugarcane borer in Louisiana. Florida Entomologist,

95: 261-267. DOI: https://doi.org/10.1653/024.095.0203.

\section{Suggestion citation:}

Sousa, JMA \& DH Matta, 2019. Controle biológico conservativo: Plantas herbáceas e a diversidade e abundância de dermápteros em algodoeiro colorido. EntomoBrasilis, 12 (3): 132-140.

Available on: doi:10.12741/ebrasilis.v12i3.841

\section{(c) (i) (2) \\ BY NC SA}
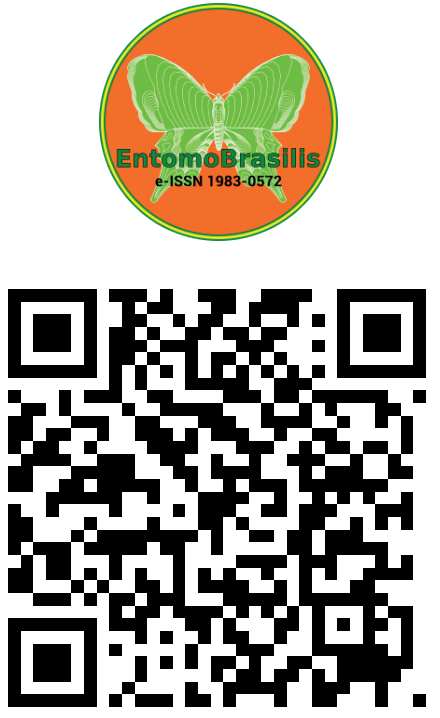